\title{
Estado da Arte da Comunicação Suplementar e/ou Alternativa: análise dos primeiros congressos brasileiros
}

\section{State of the Art of Argumentative and Alternative Communication: analysis of the first Brazilian conferences}

\begin{abstract}
Resumo: Introdução: A Comunicação Suplementar e/ou Alternativa (CSA) expandiu-se além do âmbito da Saúde, especialmente em instituições especializadas, no Brasil, configurando-se como área clínico-educacional, sendo que, desde suas origens, possuía um perfil mais assistencial, voltado, predominantemente, a crianças com paralisia cerebral. Foram realizados quatro congressos brasileiros de CSA (2005 a 2011). Interessa estabelecer um panorama, a partir do conhecimento produzido nesses eventos, considerando-se que a publicação da área é reduzida. Objetivo: I nvestigar o conhecimento científico de CSA nas publicações dos três primeiros congressos nacionais. Método: Foram analisados 182 trabalhos dos Anais (resumos) e livros (trabalhos completos) quanto à abrangência e foco das ações. Não se incluiu o quarto congresso, pois não havia ocorrido, na época da coleta de dados. Resultados: Mostram maior diversidade de usuários, patologias, faixa etária e origem institucional e geográfica dos trabalhos. Conclusão: Os resultados refletem o desenvolvimento da CSA no país, nos últimos anos.

Palavras-chave: Estudos de Linguagem. Sistemas de Comunicação Alternativos e Aumentativos. Revisão Sistemática. Educação Especial.
\end{abstract}

\begin{abstract}
Introduction: The Augmentative and Alternative Communication (AAC) has extended beyond the scope of health care, particularly at specialized institutions, in Brazil, as a clinical and educational practice. Since the beginning, the AAC presented a clinical profile attending mostly children with cerebral palsy. There were four Brazilian Conferences, from 2005 to 2011. It became interesting to establish an overview of the current state of the AAC based on the scientific production generated in those events. Objective: To analyze the AAC scientific production in the first Brazilian Conferences. Methods: 182 studies (posters and oral presentations) were analyzed regarding scope and action focus. The fourth conference was not included because it occurred after the data collection period. Results: The findings show a diversity of clients, pathologies, age, institution and geographic localization of the studies. Conclusion: The results reflected the AAC development in Brazil over the last years.

Keywords: Language Arts. Communication Aids for Disabled. Systematic Review. Special Education.

CHUN, Regina Yu Shon; MOREIRA, Eliana Cristina; DALLAQUA, Graziella Batista. Estado da Arte da Comunicação Suplementar e/ ou Alternativa: análise dos primeiros congressos brasileiros. I nformática na Educação: teoria e prática, Porto Alegre, v. 15, n. 2, p. 199-214, jul./dez. 2012.
\end{abstract}

\author{
Regina Yu Shon Chun \\ Eliana Cristina Moreira \\ Graziella Batista Dallaqua \\ Universidade Estadual de Campinas
}

\section{Introdução}

A Comunicação Suplementar e/ou Alternativa (CSA) constitui, ainda, prática pouco difundida em nosso país (CHUN, 2009), sendo que, na literatura internacional, situa-se como Augmentative and Alternative Communication (AAC), e, segundo a divisão especial da AAC na American Speech-Language-Hearing Association (ASHA), é definida da seguinte forma:

[...] AAC refers to an area of research, clinical and educational practice. AAC involves attempts to study and when necessary compensate for temporary or permanent impairments, activity limitations, and participations restrictions of person with severe disorders of speech-language production and/or comprehen- 
sion, including spoken and written modes of communication. (ASHA, 2005 apud BEUKELMAN; MIRENDA, 2007, p. 3-4)

Desde suas origens, a CSA, em nosso país, possui um perfil mais clínico, voltado, predominantemente, ao atendimento de crianças não oralizadas, com paralisia cerebral. Atualmente, expandiu-se além do âmbito da Saúde, especialmente em instituições especializadas, configurando-se como área clínico-educacional (REILY, 2007, CHUN, 2009), que visa, de forma temporária ou permanente, apoiar, complementar, suplementar/meIhorar ou substituir as formas de produção e interpretação verbal de sujeitos não falantes ou com dificuldades de linguagem (CHUN; FEDOSSE; COUDRY, 2007, versão adaptada da ASHA pelas autoras).

Como pontua Reily:

Encontramo-nos numa era em que vigora um paradigma de inclusão escolar que defende o direito à educação gratuita de qualidade para todos. [...] A despeito das críticas direcionadas contra as escolas especiais, os centros de atendimentos clínicos e as oficinas pedagógicas e ocupacionais, tais estabelecimentos tiveram um papel significativo de produção de conhecimento sobre metodologias de trabalhos com deficiências específicas. (REILY, 2007, p. 19)

Tais instituições tiveram um papel preponderante na introdução e implementação de práticas de CSA, despontando como recurso auxiliar na atuação desses profissionais, de forma a promover melhor qualidade de vida aos seus usuários.

Reily conclama essas instituições, antes que se apaguem da memória iniciativas sociais tão importantes, a “[...] investir na recuperação das narrativas pioneiras das escolas especiais e dos registros pedagógicos e clínicos de profissionais que contribuíram para a construção de um modo brasileiro de pensar metodologias de educação especial e terapêuticas [...]" (REILY, 2007, p. 20), já que são essas práticas que consolidam a atuação clínica e escolar, em nossa história recente. Diante do exposto, interessa, neste trabalho, analisar a produção científica dos primeiros Congressos Brasileiros de Comunicação Alternativa, realizados de 2005 a 2011, por se constituírem registros oficiais e pioneiros do que está sendo produzido na área, em nosso país.

Além disso, Ferreira reporta que, nas últimas décadas:

[...] tem se produzido um conjunto significativo de pesquisas conhecidas pela denominação 'estado da arte' ou 'estado do conhecimento'. Definidas como de caráter bibliográfico, elas parecem trazer em comum o desafio de mapear e de discutir uma certa produção acadêmica em diferentes campos do conhecimento, [...] (FERREI RA, 2002, p. 257).

A autora acrescenta que estes pesquisadores são movidos pelo não conhecimento daquilo que se produz em determinada área, como no caso da temática em foco.

De modo que este trabalho torna-se importante, neste momento da história da CSA, para conhecimento de sua evolução científica, pois se trata de uma área nova no Brasil, que embora tenha sofrido grande expansão, nos últimos anos, constitui ainda, prática pouco reconhecida em nosso país (CHUN, 2009). Daí, a necessidade de se conhecer as pesquisas e o conjunto de informações e resultados já obtidos de uma dada área, para que se possa organizá-los e identificar perspectivas, lacunas e vieses (SOARES, 1987 apud FERREIRA, 2002). 
Para melhor compreensão e conhecimento do que está sendo produzido em CSA, é importante resgatar aspectos históricos e conceituais. De um lado, na Saúde, os avanços tecnológicos têm possibilitado maior sobrevida de pessoas, de diferentes faixas etárias, com alterações ou disfunções neurológicas, gerando consequente aumento da demanda de assistência à saúde, bem como educacional. Assim, de outro lado, considerando-se que, "[...] toda pessoa tem direito à educação [...]" (UNESCO, 1998, p.2), impõe a mobilização de políticas, objetivando o acesso e promoção da equidade, sendo que a CSA vem se mostrando como uma abordagem capaz de proporcionar formas alternativas de comunicação e favorecimento da escolarização.

Para a ASHA (2004, 2005 apud BEUKELMAN; MIRENDA, 2007), a CSA deve ser pensada como um sistema composto de quatro componentes: símbolos de CSA (gráficos, auditivos, tácteis como sinais, gestos e expressão facial, fotos, objetos, desenhos, ortografia, etc.), dispositivos de CSA (eletrônicos ou não), técnicas de CSA (formas como as mensagens podem ser transmitidas) e estratégias de CSA (como se comunicar com mais efetividade e eficácia). De acordo com a ASHA, estes quatro itens constituem elementos cruciais nas intervenções terapêuticas e educacionais com a CSA (ASHA, 2004 apud BEUKELMAN; MIRENDA, 2007).

A aplicabilidade da CSA varia de acordo com as necessidades do usuário e disponibilidade de recursos, abrangendo as denominadas tecnologias de apoio tradicionais, como também aqueles envolvendo alta tecnologia. Tetzchner e Martinsen (2000) esclarecem que estas se constituem em pranchas confeccio- nadas com símbolos, fotos, letras do alfabeto, números, figuras e outros recursos que se mostrarem necessários. O material é disposto na prancha de comunicação, de modo a favorecer o manuseio, pelo sujeito, e possibilitar que se comunique com maior facilidade e efetividade (TREVIZOR; CHUN, 2004).

Para utilização das mesmas, o usuário pode realizar seleção direta dos símbolos, indicando o que deseja expressar com a mão, o olhar e outras formas ou movimentos que sejam mais eficientes para ele. Além disso, o processo de comunicação pode ocorrer com o auxílio de um interlocutor, que aponta/indica, pela pessoa, de modo direto ou por um sistema de varredura, símbolo a símbolo, ou por blocos de símbolos, entre as opções disponíveis, de modo que a pessoa possa transmitir a mensagem desejada.

Os recursos de alta tecnologia são mais recentes e baseiam-se em dispositivos que utilizam a tecnologia dos computadores (TETZCHNER; MARTINSEN, 2000), tornandose apoio para a comunicação de pessoas com comprometimentos variados de linguagem e motores. Existem programas (softwares) que utilizam símbolos gráficos, predição de palavras, vocalizadores, dentre outros recursos, que facilitam a comunicação, propiciando uma seleção mais rápida, além de possibilitar que as pessoas com necessidades especiais possam escrever uma carta, acionar dispositivos do ambiente, como acender a luz ou televisão, por exemplo. Tais recursos constituem relevante alternativa para fins educacionais, auxiliando no processo de letramento e de ensino/aprendizagem dos alunos com necessidades educacionais especiais.

Para a introdução da CSA, é necessária uma avaliação das necessidades do sujeito 
para identificar as demandas, determinar objetivos e delinear as propostas a serem implantadas. A complexidade de utilização dos Sistemas Suplementares e/ ou Alternativos de Comunicação (SSAC) abrange palavras isoladas, mensagens telegráficas ou frases simples a enunciados mais complexos e estão na dependência das possibilidades linguísticocognitivas do usuário e do favorecimento do uso, em seu meio, por outros interlocutores, como os membros da família, da comunidade e da equipe de assistência, seja da Saúde, seja da Educação.

Cabe ressaltar que a utilização da CSA não impede que a pessoa recupere a fala, ao contrário, seu uso pode favorecê-la. Sua abordagem, contudo, frequentemente, é associada a tarefas descontextualizadas de reconhecimento, nomeação e/ou ao mero treinamento de apontar símbolos (CHUN, 2010). Assim, assume importância outro olhar acerca da linguagem, do sujeito e dos processos de Saúde e de Educação, na atuação com a CSA. Nesse sentido, uma perspectiva de orientação discursiva possibilita investigar os processos de significação envolvidos nas atividades linguístico-cognitivas de sujeitos com comprometimentos diversos de linguagem, de forma contextualizada, buscando-se contemplar suas singularidades e compreendê-los em seus contextos histórico, social e cultural.

Ainda não há uma versão brasileira oficial da denominação Augmentative and Alternative Communication (AAC), encontrando-se denominações como Comunicação Alternativa, Comunicação Aumentativa e Ampliada (CAA) e Comunicação Suplementar e/ou Alternativa (CSA), dentre outras (CHUN, 2009). Retomamos brevemente essa questão, já discu- tida em trabalhos anteriores (CHUN, 2009), de forma a esclarecer o uso da designação Comunicação Suplementar e/ ou Alternativa e da sigla correspondente (CSA), neste artigo.

Como esclarecem Manzini e Deliberato (2006), Reily (2007) e Chun (2009), Comunicação Alternativa traz a idéia de substituição da fala, o que não se constitui como objetivo central da CSA, que seria favorecer a linguagem e a comunicação daqueles que dela necessitam. Portanto, não se recomenda a utilização isolada dessa expressão (LLOYD; KANGAS, 1988). Esses últimos autores já assinalavam, em 1988, que os países de língua não inglesa não tinham dificuldades em traduzir alternative communication (comunicação alternativa), uma vez que se encontram correlatos similares em várias línguas.

Por outro lado, Lloyd e Kangas (1988) destacavam que o mesmo não ocorre com a expressão augmentative communication, em que há dificuldades de tradução de modo a manter o sentido original. Os autores apontam um problema de definição e de lógica nesse caso. Para Manzini e Deliberato (2006), Reily (2007) e Chun (2009), as expressões comunicação suplementar ou comunicação ampliada remetem à idéia de ser suplementar à fala.

Como explica Reily,

[...] o termo aumentativo não existe no dicionário em português, e se fosse o caso de criar um neologismo, tal termo não seria apropriado, a nosso ver, porque não dá conta do sentido de augmentative, que traz a conotação de auxiliar, servir como apoio, complementar, enfim suplementar à comunicação de outros meios já empregados como os gestos, o olhar, a expressão facial, o sorriso e mesmo as alterações de tônus muscular além da própria fala ou vocalização, que pode estar presente. (REILY, 2007, p. 28) 
Sendo assim, a CSA vai além de substituir a fala como um sistema alternativo, ela, por si só, compõe o significado, uma vez que a linguagem, apoiada pelos recursos da CSA, é colocada em movimento pelo outro (REILY, 2007).

A CSA vem se expandindo no Brasil, desde os trabalhos pioneiros, no final dos anos de 1970, em São Paulo e no Rio de Janeiro, em função do empenho de profissionais da área e do trabalho desenvolvido, em seus primórdios, em clínicas e instituições especializadas. Atualmente, o campo da CSA se ampliou, além do âmbito de desses espaços especializados, particularmente no contexto educacional.

Em consonância com as atuais políticas inclusivas, que preconizam escola para todos e CSA como um dos recursos educacionais especiais (BRASIL, 2005, MANZINI; DELIBERATO, 2006), o uso da CSA tem se expandido no ambiente escolar, especialmente por meio de ações das prefeituras municipais de várias cidades brasileiras, através das Secretarias de Educação e de Saúde, favorecendo a comunicação e a inclusão dos alunos com necessidades educacionais especiais.

Como esclarecem Manzini e Deliberato (2006), em publicação da Secretaria de Educação Especial do Ministério da Educação (MEC), os recursos de CSA são um meio eficaz para garantir a inclusão de alunos com necessidades educacionais especiais na área da comunicação, no processo de interação entre professor e aluno, de modo que o educando possa se "[...] comunicar com as outras pessoas e expor suas idéias, pensamentos e sentimentos se puder utilizar recursos especialmente desenvolvidos e adaptados para o meio no qual está inserido" (MANZI-
NI; DELIBERATO, 2006, p.4). Além de que, dentre as políticas estabelecidas pelo MEC, para escolas inclusivas, recomenda-se “[...] propiciar os melhores níveis de comunicação e interação [...]" e "[...] adotar sistemas de comunicação alternativos para os alunos impedidos de comunicação oral [...]" (BRASIL, 2005, p.71).

Vitaliano (2007) coloca que diversas transformações vêm ocorrendo no sistema de educação brasileiro, em prol de mudanças que proporcionem uma política inclusiva, com vistas ao atendimento educacional especializado gratuito, aos alunos com necessidades especiais, preferencialmente, na rede regular de ensino. A educação como parte de um conjunto de direitos sociais, previstos pela Constituição Brasileira, visa garantir a diminuição da desigualdade entre os membros da sociedade (TAKASE; CHUN, 2010).

Além dessa questão, é importante considerar o significativo desenvolvimento e crescimento de pesquisas de CSA no meio acadêmico (REILY, 2007, NUNES, L.R.; NUNES, D.R., 2007, CHUN, 2009). Os aspectos até aqui colocados se refletem nos Congressos de Comunicação Alternativa, realizados em nosso país, e justificam investigações para o conhecimento dessa realidade.

Zangari, Loyd e Vicker (1994), em clássica revisão da CSA, no periódico Augmentative and Alternative Communication, publicação especializada da área, pontuam que, apesar da história ser construída a cada dia, somente poderá ser lembrada e avaliada se houver registros escritos disponíveis. Os autores acrescentam que muito se fez nesse curto período da CSA, no mundo, porém, muitas pessoas ainda desconhecem esse trabalho e a sua evolução. Embora antigo, reflete a si- 
tuação atual em nosso país, reafirmando a importância de estudos, como aqui proposto, com vistas a traçar um panorama do estado da arte da CSA, no Brasil, no período de realização dos seus primeiros congressos, de 2005 a 2011.

Apesar do crescimento da CSA no cenário nacional, há somente dois estudos de revisão publicados (BERBERIAN et al., 2009, CHUN, 2009), que mostram a reduzida produção em periódicos especializados. Segundo esses autores, até 2005, havia apenas 11 artigos indexados em Fonoaudiologia e 16, até 2007, nas bases de dados LILACS e SCIELO, e nenhum na MEDLINE. Em novo levantamento correspondente ao período de 1996 a julho de 2011, encontramos um total de 51 publicações.

Em 2004, na 11ạ Conferência Bienal da International Society for Augmentative and Alternative Communication (ISAAC), em Natal (Rio Grande do Norte, Brasil), planejouse o primeiro evento de CSA em nosso país. Assim, em 2005, foi realizado o I Congresso Brasileiro de Comunicação Alternativa - denominação que tem se mantido em suas edições até 2011. Foram realizados quatro encontros nacionais até esse ano. A análise da produção científica desses eventos possibilita um recorte da prática em CSA, em nosso país.

Como pontuam Zangari, Loyd e Vicker (1994), muitas são as dificuldades para se desenvolver um panorama histórico da CSA, dentre elas, os diversos trabalhos e experiências não documentados. É importante considerar também, como assinalam esses autores, que o presente, de algum modo, começa no passado, e refletir sobre as experiências do passado constitui um ponto fundamental para planejar novas intervenções e ir adiante. Berberian et al. (2009) e Chun et al. (2010) também pontuam que é essencial a participação e análise das áreas implicadas na CSA.

De modo que a investigação da produção desse campo possibilita mapear o conhecimento produzido nos congressos nacionais como subsídio para o delineamento do estado da arte da CSA, em nosso país, bem como para maior eficácia das intervenções de Saúde e Educação, nesse campo, em uma perspectiva de atenção integral e de inclusão social. Diante do exposto, justifica-se a necessidade de maior investimento em pesquisas nacionais e internacionais de revisão sobre a área, como aqui proposto.

\section{Objetivo}

Este artigo tem como objetivo investigar a produção do conhecimento em Comunicação Suplementar e/ou Alternativa nas publicações (Anais e livros) dos primeiros congressos brasileiros da área.

\section{Método}

O estudo foi desenvolvido no Centro de Estudos, Pesquisa e Reabilitação Prof. Dr. Gabriel O. S. Porto (CEPRE) da Faculdade de Ciências Médicas da Universidade Estadual de Campinas.

Esta pesquisa, de caráter bibliográfico, tem como foco os três primeiros Congressos Brasileiros de Comunicação Alternativa, sem a pretensão de ser uma revisão completa, mas, 
sim, um retrato parcial da história do desenvolvimento dessa nova área do conhecimento.

Foram considerados para a coleta de dados os materiais disponibilizados nos encontros realizados em 2005, 2007 e 2009, isto é, os resumos e/ou trabalhos completos das sessões de comunicação oral ou pôster, alguns em forma impressa, outros em formato digital, além dos livros publicados (trabalhos completos selecionados para publicação) referentes aos eventos. Um total de 182 trabaIhos foi analisado. O IV Congresso Brasileiro de Comunicação Alternativa, ocorrido em 2011, no Rio de Janeiro, não foi incluído, pois sua realização se deu após o período de coleta de dados.

O critério de escolha da análise dos trabalhos das sessões de comunicação oral e pôsteres dos congressos decorre do fato que, nesse espaço, várias são as experiências relatadas, além de apresentar resultados de pesquisas acadêmicas.

Considerando-se os dados históricos da CSA, em nosso país (REILY, 2007, CHUN, 2009), tais como as práticas iniciais serem voltadas a crianças com paralisia cerebral e desenvolvidas principalmente em instituições especializadas, as principais questões relativas à produção de conhecimento, na área, que motivaram esta pesquisa, foram:

I - Quais são, atualmente, os usuários de CSA? A que faixa etária pertencem?

II - Onde os trabalhos de CSA vêm sendo desenvolvidos, tanto em relação ao vínculo institucional quanto à localização geográfica?

Para a coleta e análise dos dados, todos os resumos e trabalhos completos disponíveis foram lidos e dispostos em uma tabela do Excel, segundo as categorias de análise estabelecidas.
Após leitura do material e de acordo com os objetivos do estudo, foram estabelecidos, como categorias de análise, os itens que se seguem: a) usuários; b) população alvo; c) área do trabalho; d) tipo de vínculo institucional dos autores e e) localidade (estado do país) onde o trabalho foi desenvolvido.

De modo que, no item usuários, os trabalhos foram, inicialmente, categorizados em usuários e não usuários. Na categoria usuários, foram estabelecidas subcategorias por ciclos de vida, classificadas em: criança, adolescente, adulto, criança e adolescente, adolescente e adulto, e todas as faixas etárias. Na categoria não usuários, considerou-se as subcategorias educadores, alunos de graduação da área da Saúde e/ ou Educação e profissionais (outros profissionais, além dos educadores). Outrossim, algumas pesquisas que não envolviam uma população específica, como os de revisão da literatura, foram consideradas como não se aplica e aquelas que não traziam informação acerca desse aspecto foram agrupadas como não especificado.

Quanto à população alvo, foi classificada por tipos de patologias e quadros dos usuários de CSA, a saber: paralisia cerebral associada ou não a outra deficiência, transtorno global do desenvolvimento e outras do espectro autista, afasia, outros transtornos neurológicos e síndromes (incluindo-se síndrome de Down, deficiência física e/ou múltipla, surdocegueira associada ou não a outras deficiências, apraxia de fala e deficiência mental) e outros quadros clínicos (incluindose deficientes auditivos, traqueostomia, deficiência visual/ baixa visão, deficiente físico e atraso no desenvolvimento).

Além disso, quando as ações eram destinadas a não usuários foram categorizadas 
em: familiares de usuários de CSA, graduandos da área de Educação e/ou Saúde, profissionais da área da Educação e/ou Saúde, sendo que os de revisão da literatura foram incluídos no grupo não se aplica.

Os estudos foram categorizados por área em duas grandes classes: Saúde e Educação.

Em relação ao vínculo institucional dos autores foram estabelecidas as seguintes categorias: universidade, centro especializado, prefeitura e universidade e centro especializado e prefeitura e centro especializado, quando havia parceria entre as instituições de origem, além de não especificado, quando não havia esse dado nos registros.

Para levantamento da localização geográfica, considerou-se o local de origem onde foi desenvolvido, tendo sido estabelecidas as subcategorias - estado do Brasil, outros países e não informado, conforme discriminado nos Anais.

Nesta fase do estudo, os dados foram submetidos à análise estatística descritiva.

\section{Resultados e Discussão}

Neste artigo, são apresentados os achados referentes às categorias descritas anteriormente. O total de apresentações no I Congresso Brasileiro de Comunicação Alternativa e I Congresso Internacional de Linguagem e Comunicação da Pessoa com Deficiência, realizado no Rio de Janeiro, em 2005, foi de 81. No entanto, somente 73 envolviam a temática Comunicação Suplementar e/ou Alternativa, de modo que os demais foram excluídos da análise. Os Anais do II Congresso Brasileiro de Comunicação Alternativa, realizado em Campinas, em 2007, continham apenas 51 trabalhos, dos quais 34 foram apresentados em forma de comunicação oral e 17 como pôsteres. No III Congresso Brasileiro de Comunicação Alternativa, realizado em São Paulo, em 2009, o registro digital continha apenas 58 comunicações orais e nenhum pôster, portanto, não pôde ser incluído nesta análise.

\section{a) Resultados relativos aos usuários}

Os achados mostram que no primeiro congresso, $90 \%$ dos trabalhos envolviam usuários; no segundo, $55 \%$ focavam os usuários e, no terceiro, $48 \%$ voltavam-se aos usuários de Comunicação Suplementar e/ou Alternativa.

Tais resultados reforçam a importância da assistência aos usuários de CSA, sendo que a reabilitação é considerada como importante estratégia de Saúde Pública, nas atuais políticas de Saúde voltadas às alterações neurológicas (WORLD HEALTH ORGANIZATION, 2006). Segundo a Organização Mundial de Saúde (OMS), o número de Disability Adjusted Life Years (DALYs) (WORLD HEALTH ORGANIZATION, 2011), ou seja, da soma dos anos de vida perdidos em função de mortalidade prematura ou de anos de vida produtiva perdidos por incapacidade, dos países em desenvolvimento, é sete vezes o de países desenvolvidos, impondo que medidas e ações sejam tomadas.

Em relação aos ciclos de vida dos usuários, os resultados, apresentados na Tabela 1, demonstram que no I Congresso, a população de crianças e adolescentes somadas chega a $71 \%$, categoria que permanece como a maioria nos eventos estudados, atingindo $61 \%$ e $68 \%$ no II e III Congressos, respectivamen- 
te. Já as apresentações direcionadas ao adulto representam somente $12 \%$ das apresentações do primeiro congresso, com significativo crescimento nos outros anos, 32\% no segundo e $25 \%$ no terceiro. Tais resultados são similares aos encontrados por Berberian et al. (2009), em revisão de artigos nacionais de CSA.

TABELA 1 - Distribuição dos Usuários Quanto aos Ciclos de Vida, por Congresso

\begin{tabular}{|c|c|c|c|c|}
\hline & $\begin{array}{c}\text { I Congres- } \\
\text { so 2005 } \\
(\%)\end{array}$ & $\begin{array}{c}\text { II Con- } \\
\text { gresso } \\
2007(\%)\end{array}$ & $\begin{array}{c}\text { III Con- } \\
\text { gresso } \\
2009 \\
(\%)\end{array}$ & $\begin{array}{c}\text { TOTAL } \\
\text { GERAL }\end{array}$ \\
\hline Crianças & 29 & 21 & 43 & $\mathbf{3 0 \%}$ \\
\hline Adolescentes & 10 & 36 & 21 & $\mathbf{1 9 \%}$ \\
\hline Adultos & 12 & 32 & 25 & $\mathbf{2 0 \%}$ \\
\hline $\begin{array}{c}\text { Crianças e } \\
\text { adolescentes }\end{array}$ & 30 & 4 & 4 & $\mathbf{1 8 \%}$ \\
\hline $\begin{array}{c}\text { Adolescentes } \\
\text { e adultos }\end{array}$ & 2 & 0 & 0 & $\mathbf{1} \%$ \\
\hline $\begin{array}{c}\text { Todas as fai- } \\
\text { xas etárias }\end{array}$ & 17 & 7 & 7 & $12 \%$ \\
\hline
\end{tabular}

FONTE: Elaborado pelas próprias autoras

Os resultados envolvendo não usuários representam, no computo geral, uma maioria de $62 \%$ na subcategoria profissionais, evidenciando uma preocupação em abranger não apenas os usuários de CSA como também outras categorias como os profissionais de saúde, educadores e alunos da graduação das áreas da Saúde e da Educação. Além disso, $10 \%$ da categoria não se aplica, evidenciam um olhar a outras questões, tais como a discussão de temas como revisão da literatura de apresentações com a CSA.

De um lado, os achados mostram que as pesquisas, majoritariamente, envolvem os usuários, ao longo dos três eventos estudados, refletindo a preocupação dos profissio- nais com os sujeitos não oralizados ou com dificuldades de linguagem foco de sua atuação. De outro, mostram que vem ocorrendo uma ampliação do campo de pesquisa com a CSA, envolvendo, também, os não usuários, a interação entre eles, levantamento da percepção dessas pessoas sobre a área, além de pesquisas sobre tecnologias de CSA.

\section{b) Resultados relativos à população} alvo

Em relação à população alvo (vide Gráfico 1), observa-se que a categoria de maior foco das ações corresponde aos usuários com paralisia cerebral associada ou não a outra deficiência (36\%, 49\% e $27 \%$, respectivamente Congressos I, II e III) e, em menor índice, a outros transtornos neurológicos e síndromes. Resultados também encontrados por Berberian et al. (2009), em que a paralisia cerebral se constitui como o quadro clínico da maioria absoluta dos estudos de CSA, publicados de 1997 a 2005.

\section{GRÁFICO 1 - Distribuição dos Trabalhos por População Alvo, por Congresso}

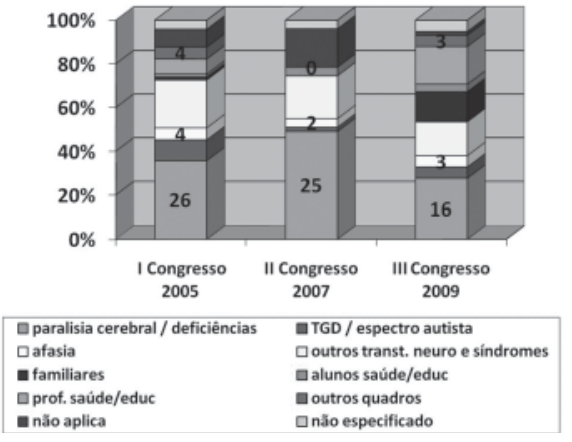

FONTE: Elaborado pelas próprias autoras

No entanto, diferentemente dos achados dessas autoras, nos três congressos encontram-se trabalhos voltados a outros quadros 
clínicos e patologias como afasias e transtornos globais de desenvolvimento (TGD) e outros, do espectro autista, população alvo de publicações posteriores a esta revisão, como os de Walter e Almeida (2010), Débora Nunes e Nunes Sobrinho (2010) e Chun (2010).

Ao longo dos congressos, observa-se um crescimento das apresentações envolvendo familiares de usuários de CSA: no primeiro (2005), foi identificada somente uma pesquisa, envolvendo exclusivamente a família (1,36\%); no segundo (2007), não houve nenhuma, e, no terceiro (2009), essa porcentagem correspondeu a $14 \%$.

Nos grupos sociais onde existem sujeitos com deficiência, é de extrema importância a intervenção com os interlocutores, pois as oportunidades de desenvolvimento de habilidades no cotidiano serão mais aproveitadas se uma comunicação efetiva ocorrer com os atores envolvidos no processo de ensino, assim como também a inclusão.

Nessa direção, Serapompa e Maia (2006) destacam que a crença, de pais e professores, no desenvolvimento do aluno e o diálogo, estabelecido entre os profissionais, a família e a criança, favorecem o desenvolvimento de situações de comunicação. Essas autoras, dentre outras, pontuam a importância dos pais ou familiares atuarem como parceiros ativos no processo de tomada de decisão e planejamento da educação de seus filhos, contribuindo para favorecer a integração escolar e social das crianças com necessidades especiais.

c) Resultados relativos à área do trabalho - Saúde ou Educação

Quanto à área do trabalho, os achados indicam que a diferença entre Saúde e Educa- ção é pequena, no decorrer dos anos, como demonstra o gráfico comparativo dos três congressos (vide Gráfico 2).

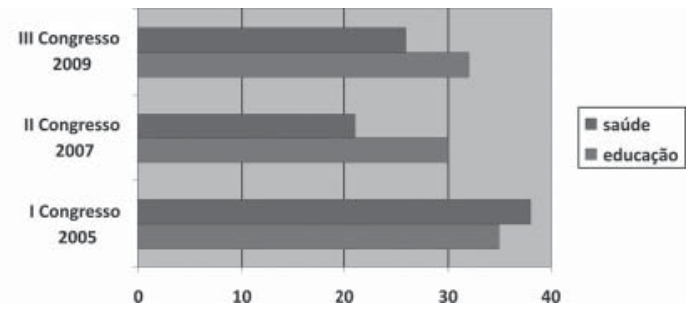

GRÁFICO 2 - Distribuição dos Trabalhos por Área Saúde e Educação, por Congresso

FONTE: Elaborado pelas próprias autoras

Tais resultados remetem a Reily, que aborda o valor da atuação conjunta entre profissionais da Saúde e educadores, considerando que discussões multiprofissionais “[...] geram ricos espaços para elaboração de princípios de trabalho e práticas que se constituem a partir de vários pontos de vista [...]" (REILY, 2007, p. 20), favorecendo a comunicação e o processo de inclusão dos alunos com necessidades educacionais especiais.

Sabe-se que a comunicação é indispensável ao ser humano e, nesse sentido, a autora pontua que uma pessoa com comprometimento da linguagem, oral ou escrita, poderá ter maior dificuldade no processo de ensino e aprendizagem, destacando o papel da CSA no âmbito da inclusão escolar.

\section{d) Resultados relativos ao vínculo ins- titucional}

Em relação ao vínculo institucional dos autores (vide Tabela 2), verifica-se que as ações se ampliam além do âmbito clínico e de centros especializados, como ocorria nas 
origens da CSA, em nosso país (REILY, 2007, CHUN, 2009), envolvendo universidades, as quais, associadas a centros especializados, constituem a maioria dos trabalhos nos três congressos (55\%, 92\% e 70\%, nos I, II e III Congressos, respectivamente).

A porcentagem de participação dos Centros Especializados, em parceria com a própria Universidade ou Secretarias da Prefeitura, em grande parte da Educação, permanece nos três congressos, porém, com atuação mais expressiva no primeiro. Nota-se um número grande de registros nos Anais dos Congressos sem a informação do vínculo institucional dos autores.

Os achados encontrados levantam a possibilidade de maior preocupação com o desenvolvimento científico da CSA, em nosso país.

TABELA 2 - Distribuição dos Trabalhos Quanto ao Vínculo Institucional, por Congresso

\begin{tabular}{|c|c|c|c|}
\hline & $\begin{array}{c}\text { I Con- } \\
\text { gresso } \\
\mathbf{2 0 0 5} \\
(\%)\end{array}$ & $\begin{array}{c}\text { I I Con- } \\
\text { gresso } \\
\mathbf{2 0 0 7} \\
\mathbf{( \% )}\end{array}$ & $\begin{array}{c}\text { I I I Con- } \\
\text { gresso } \\
\mathbf{2 0 0 9} \\
\mathbf{( \% )}\end{array}$ \\
\hline Universidade & 44 & 72 & 60 \\
\hline $\begin{array}{c}\text { Universidade e Cen- } \\
\text { tro Especializado }\end{array}$ & 11 & 20 & 10 \\
\hline Centro Especializado & 34 & 4 & 2 \\
\hline $\begin{array}{c}\text { Centro Especializado } \\
\text { e Prefeitura }\end{array}$ & 7 & 0 & 2 \\
\hline Prefeitura & 4 & 4 & 5 \\
\hline Não informado & 0 & 0 & 21 \\
\hline
\end{tabular}

FONTE: Elaborado pelas próprias autoras

\section{e) Resultados relativos ao local de origem dos trabalhos}

A categoria local de origem traz, em seus resultados, um panorama nacional quanto à região (estado do Brasil) onde foi desenvolvido o trabalho, a Tabela 3 mostra essa dis- tribuição, incluindo outros países no I Congresso.

TABELA 3 - Distribuição dos Trabalhos por Local de Origem, por Congresso

\begin{tabular}{|c|c|c|c|}
\hline & $\begin{array}{c}\text { I Con- } \\
\text { gresso } \\
\mathbf{2 0 0 5}(\%)\end{array}$ & $\begin{array}{c}\text { I I Con- } \\
\text { gresso } \\
\mathbf{2 0 0 7}(\%)\end{array}$ & $\begin{array}{c}\text { I I Con- } \\
\text { gresso } \\
\mathbf{2 0 0 9}(\%)\end{array}$ \\
\hline São Paulo & 44 & 78 & 48 \\
\hline Rio de Janeiro & 27 & 6 & 21 \\
\hline Minas Gerais & 1,5 & 0 & 0 \\
\hline Espírito Santo & 4 & 0 & 0 \\
\hline $\begin{array}{c}\text { Rio Grande do } \\
\text { Sul }\end{array}$ & 8 & 8 & 2 \\
\hline Paraná & 0 & 0 & 3 \\
\hline Santa Catarina & 1,5 & 0 & 0 \\
\hline Bahia & 1,5 & 0 & 0 \\
\hline Sergipe & 0 & 0 & 2 \\
\hline Pernambuco & 1,5 & 0 & 0 \\
\hline Pará & 1,5 & 4 & 2 \\
\hline $\begin{array}{c}\text { São Paulo e Rio } \\
\text { Janeiro }\end{array}$ & 0 & 2 & 0 \\
\hline $\begin{array}{c}\text { São Paulo e } \\
\text { Brasília }\end{array}$ & 1,5 & 0 & 0 \\
\hline Não Informado & 3 & 2 & 22 \\
\hline Outros países & 5 & 0 & 0 \\
\hline
\end{tabular}

FONTE: Elaborado pelas próprias autoras

Nota-se a predominância dos dados referentes aos estados da região Sudeste: São Paulo, Rio de Janeiro, Espírito Santo e Minas Gerais. Estes resultados são similares aos encontrados por Berberian et al. (2009), em relação à procedência geográfica dos autores, sendo que São Paulo representou cerca de $61,1 \%$ dos trabalhos publicados, no período estudado pelas autoras, mostrando uma concentração da produção científica na região Sudeste, assim como em nosso estudo. 


\section{Considerações Finais}

Este trabalho, de revisão da produção científica dos três primeiros Congressos Brasileiros de Comunicação Alternativa, teve como finalidade traçar um retrato parcial da história do desenvolvimento dessa área, em nosso país, por meio do mapeamento da produção disponível e, como tal, pode ser considerada representativa do estado da arte desse contexto. Não houve a pretensão de esgotar o assunto, mas sim, sistematizar a informação disponível, uma vez que há necessidade de maior produção de conhecimentos sobre a área.

A riqueza das experiências mereceria uma análise mais detalhada, mas nem sempre isso foi possível, pela falta de dados completos. Por outro lado, os resultados possibilitaram chegar a conclusões importantes, que mostram a evolução da Comunicação Suplementar e/ou Alternativa, no Brasil, no período estudado, como evidenciado na produção analisada.

A CSA, como colocado na I ntrodução deste artigo, teve origem essencialmente clínica e reabilitadora, no cenário nacional, assumindo uma abordagem mais de Saúde, perfil que se mantém, em grande parte dos trabaIhos, ao mesmo tempo em que os achados evidenciam o crescimento e a abrangência na Educação, reafirmando que ambas as áreas são fundamentais no processo de melhoria da qualidade de vida da população com deficiência.

Além disso, mostram o valor da atuação conjunta entre profissionais da Saúde e educadores, em que a troca de experiências e discussões constitui rico espaço de reflexão e construção de saberes multidisciplinares de abordagens e pontos de vistas distintos.

Outra questão que desponta na análise é que a maioria das ações volta-se, ainda, a usuários, crianças e/ou adolescentes, com paralisia cerebral (PC), associada ou não a outras deficiências, nos primeiros congressos, mantendo-se essa população alvo ao longo dos demais anos, porém, abrangendo também, outras faixas etárias. Verifica-se, portanto, que se mantém como patologia de maior foco das ações, mas se ampliam os ciclos de vida, atingindo não apenas crianças como historicamente vinha ocorrendo.

Os achados demonstram maior abrangência dos trabalhos no decorrer dos congressos, voltando-se a usuários com outras patologias e síndromes diversas (Angelman, síndrome de Down e outras), transtornos globais do desenvolvimento e outras do espectro autista, afasia e surdo-cegueira, além de múltiplas deficiências.

Além disso, observa-se o desenvolvimento de ações voltadas a não usuários de CSA, quer sejam familiares, profissionais de Educação e outros, como estudantes de graduação.

Destaca-se, nesse sentido, o valor da parceria com os familiares e da atuação multi e interdisciplinar nas áreas da Saúde e da Educação, abrangendo tanto os profissionais como os estudantes de graduação, tendo em vista maior efetividade e um olhar integral das ações.

Verifica-se que os estudos se ampliam além do âmbito clínico e de centros especializados, envolvendo universidades, prefeituras e parcerias entre universidades e os próprios centros especializados, além de ter havido expansão dos trabalhos na área da Educação. 
Assim, observa-se um decréscimo dos trabaIhos realizados apenas por clínicas ou centros especializados, que passam a desenvolver parcerias com as universidades e/ou prefeituras, ao longo dos anos. Observa-se, também, um crescimento das pesquisas acadêmicas, o que parece evidenciar o maior interesse e desenvolvimento científico da CSA, nos eventos analisados, necessário para a consolidação e fortalecimento da área, em nosso país.

Além disso, nota-se um pequeno aumento da proporção de estudos na área de Educação em relação à Saúde, embora os índices isolados por área (Educação e Saúde) não sofram muita variação nos três congressos analisados.

Tais resultados confirmam o que se observa na literatura, de que a CSA, no Brasil, vem ganhando maior configuração clínicoeducacional, o que, por sua vez, constitui em fator que favorece a inclusão dos alunos com necessidades especiais, dada a expansão da CSA, no âmbito educacional.

As origens da CSA, no cenário nacional, ocorreram nas cidades de São Paulo e Rio de Janeiro e, posteriormente, Belo Horizonte, sendo que se verifica que a maior concentração dos trabalhos permanece na região Sudeste do país.

Os achados possibilitam traçar um panorama do estado da arte da Comunicação
Suplementar e/ou Alternativa, nos primeiros congressos brasileiros, evidenciando um crescimento geral e maior abrangência da população alvo e das ações desenvolvidas, particularmente no âmbito educacional. Verifica-se maior expansão e diversidade da produção científica e desenvolvimento da área com vistas à atenção integral, desenvolvimento de intervenções mais eficazes e consequente favorecimento da linguagem, equiparação de oportunidades, inclusão social e qualidade de vida das populações que necessitam dos recursos da CSA.

Cabe destacar, como limites do estudo, o fato de que nem todos os trabalhos estão contemplados nos Anais, bem como as informações constantes são diferentes de um congresso para outro e, outras vezes, faltam dados em relação às categorias de interesse de estudo. Por exemplo, um aspecto interessante para análise, dado o caráter multi e interdisciplinar desse campo, seria a caracterização dos profissionais envolvidos nas ações e pesquisas, mas a maior parte dos registros não contempla essa informação.

Por fim, os resultados mostram avanços na produção científica em CSA, no Brasil, porém, ainda há necessidade de maior investimento de estudos de revisão, nacionais e internacionais, na área de comunicação alternativa.

\section{Referências}

BERBERIAN, A.P. et al. A Produção do Conhecimento em Fonoaudiologia em Comunicação Suplementar e/ ou Alternativa: análise de periódicos. Revista CEFAC, São Paulo, v. 11, supl. 2, p. 258-266, 2009. 
BEUKELMAN, D.R.; MIRENDA, P. Augmentative and Alternative Communication: Supporting Children and Adults with Complex Communication Needs. 3. ed. Baltimore: Paul H. Brookes Pub., 2007.

BRASIL. Ministério da Educação. Secretaria da Educação Especial. Saberes e Práticas de Inclusão: recomendações para a construção de escolas inclusivas. Brasília, 2005.

CHUN, R.Y.S. Comunicação Suplementar e/ou Alternativa: abrangência e peculiaridades dos termos e conceitos em uso no Brasil. Pró-Fono: revista de atualização científica, São Paulo, v. 21, p. 69-74, 2009.

CHUN, R.Y.S. Processos de Significação de Afásicos Usuários de Comunicação Suplementar e/ou Alternativa. Revista da Sociedade Brasileira de Fonoaudiologia, São Paulo, v. 15, n. 4, p. 598-603, 2010.

CHUN, R.Y.S. et al. AAC Overview: Analysis of Scientific Production of ISAAC Brazil Conferences: chapter in the making. In: BIENNIAL CONFERENCE OF THE INTERNATIONAL SOCIETY FOR AUGMENTATIVE AND ALTERNATIVE COMMUNICATION (ISAAC), 14., 2010, Barcelona. Proceedings. Barcelona: [s.n.], 2010. p. 257.

CHUN, R.Y.S.; FEDOSSE, E.; COUDRY M.I.H. Avaliação e Acompanhamento Fonoaudiológico de Sujeitos não Falantes. Diretrizes, Normas e Condutas - Área da Saúde, 2007. Disponível em: <http://www.fcm. unicamp.br/diretrizes/temas.html>. Acesso em: 08 set. 2011.

FERREIRA, N.S. de A. As Pesquisas Denominadas "Estado da Arte". Educação e Sociedade, São Paulo, ano 23, n. 79, p. 257-272, 2002.

LLOYD, L. L.; KANGAS, K. AAC Terminology Policy and Issues Update. Augmentative and Alternative Communication, Baltimore, v. 4, n. 1, p. 167-70, 1988.

MANZINI, E.J .; DELIBERATO, D. Portal de Ajudas Técnicas Para a Educação: equipamento e material pedagógico especial para educação, capacitação e recreação da pessoa com deficiência física: recursos para comunicação alternativa. Brasília: MEC/SEESP, 2006.

NUNES, D.R. de P.; NUNES SOBRINHO, F. de P. Comunicação Alternativa e Ampliada Para Educandos com Autismo: considerações metodológicas. Revista Brasileira de Educação Especial, Marília, v. 16, n. 2, p. 297312, 2010.

NUNES, L.R. d'O. de P.; NUNES, D.R. de P. Um Breve Histórico da Pesquisa em Comunicação Alternativa na UERJ. In: NUNES, L.R.; PELOSI, M.B.; GOMES, M.R. (Org.). Um Retrato da Comunicação Alternativa no Brasil: relatos de pesquisas e experiências. Rio de Janeiro: Ed. Quatro Pontos, 2007. v. 1, p.19-32.

REILY, L. Sobre Como o Sistema Bliss de Comunicação Foi Introduzido no Brasil, Divulgado Entre Profissionais que Trabalhavam com Pessoas Não Falantes ou sem Fala Funcional, Posteriormente Ganhando Adeptos 
e Críticos que Buscaram Outras Opções de Sistemas Pictográficas e como a Informática Veio a Contribuir Para a Autonomia dos Usuários de Modo que Ele Pudessem Constituir-se Como Cidadãos de Escrita Autônoma. In: NUNES, L.R.; PELOSI, M.B.; GOMES, M.R. (Org.). Um Retrato da Comunicação Alternativa no Brasil: relatos de pesquisas e experiências. Rio de Janeiro: Ed. Quatro Pontos, 2007. v. 2, p.19-45.

SERAPOMPA, M.T.; MAIA, S.M. Acolhimento e Inclusão: da clínica ao acompanhamento escolar de um sujeito com Síndrome de Down. Distúrbios da Comunicação, São Paulo, v. 18, n. 3, p. 313-322, 2006.

TAKASE, E.M.; CHUN, R.Y.S. Comunicação e Inclusão de Crianças com Alterações de Linguagem de Origem Neurológica na Perspectiva de Pais e Educadores. Revista Brasileira de Educação Especial, Marília, v. 16, n. 2, p. 251-264, 2010.

TETZCHNER, S.V.; MARTINSEN, H. Introdução à Comunicação Aumentativa e Alternativa. Porto: Porto Ed., 2000.

TREVIZOR, T.T.; CHUN, R.Y.S. O Desenvolvimento da Linguagem por Meio do Sistema Pictográfico de Comunicação. Pró-Fono: revista de atualização científica, São Paulo, v. 16, n. 3, p. 323-332, 2004.

UNESCO. Declaração Mundial Sobre Educação Para Todos: satisfação das necessidades básicas de aprendizagem, Jomtien, 1990. [S.I.], 1998.

VITALIANO, C.R. Análise das Necessidades da Preparação Pedagógica de Professores de Licenciatura Para Inclusão de Alunos com Necessidades Educacionais Especiais. Revista Brasileira de Educação Especial, Marília, v. 13, n. 3, p. 399-414, 2007.

WALTER, C.; ALMEIDA, M.A. Avaliação de um Programa de Comunicação Alternativa e Ampliada Para Mães de Adolescentes com Autismo. Revista Brasileira de Educação Especial, Marília, v. 16, n. 3, p. 429-446, 2010.

WORLD HEALTH ORGANIZATION. Neurological Disorders: Public Health Challenges. Switzerland, 2006.

WORLD HEALTH ORGANIZATION. DALYS/YLDs Definition. [S.I., 2011?]. Disponível em: <http://www.who. int/mental_health/management/depression/daly/en/index.html> Acesso em: 04 dez. 2012.

ZANGARI, C.; LOYD, L.L.; VICKER, B. Augmentative and Alternative Communication: an historic perspective. Augmentative and Alternative Communication, Baltimore, v. 10, n. 1, p. 27-59, 1994. 


\section{Regina Yu Shon Chun}

Fonoaudióloga, docente do Curso de Fonoaudiologia e do Mestrado Saúde, Interdisciplinaridade e Reabilitação da Faculdade de Ciências Médicas - FCM/ UNICAMP - Universidade Estadual de Campinas - Campinas - SP/Brasil. Email: reginayu@fcm.unicamp.br.

\section{Eliana Cristina Moreira}

Fonoaudióloga, Mestre em Saúde da Criança e do Adolescente da FCM/UNICAMP - Universidade Estadual de Campinas Campinas - SP/Brasil. E-mail: elianacmoreira@gmail.com.

\section{Graziella Batista Dallaqua}

Fonoaudióloga, aluna do Mestrado Saúde, Interdisciplinaridade e Reabilitação da FCM/ UNICAMP - Universidade Estadual de Campinas - Campinas - SP/Brasil. E-mail: gradallaqua@yahoo.com.br. 